\title{
Computerized decision support for beneficial home-based exercise rehabilitation in patients with cardiovascular disease
}

\author{
Andreas Triantafyllidis ${ }^{\mathrm{a}, \mathrm{b}, *}$, Dimitris Filos ${ }^{\mathrm{a}, \mathrm{b}}$, Roselien Buys ${ }^{\mathrm{c}, \mathrm{d}}$, Jomme Claes ${ }^{\mathrm{c}}$, \\ Véronique Cornelissen ${ }^{\mathrm{d}}$, Evangelia Kouidi ${ }^{\mathrm{e}}$, Anargyros Chatzitofis ${ }^{\mathrm{f}}$, Dimitris Zarpalas, \\ Petros Daras ${ }^{\mathrm{f}}$, Deirdre Walsh ${ }^{\mathrm{g}}$, Catherine Woods ${ }^{\mathrm{h}}$, Kieran Moran ${ }^{\mathrm{g}}$, Nicos Maglaveras ${ }^{\mathrm{a}, \mathrm{b}}$, \\ Ioanna Chouvarda ${ }^{\mathrm{a}, \mathrm{b}}$ \\ a Institute of Applied Biosciences, Centre for Research and Technology Hellas, Greece \\ ${ }^{\mathrm{b}}$ Lab of Computing, Medical Informatics and Biomedical Imaging Technologies, School of Medicine, Aristotle University of Thessaloniki, Greece \\ ${ }^{c}$ Department of Cardiovascular Sciences, KU Leuven, Belgium \\ ${ }^{\mathrm{d}}$ Department of Rehabilitation Sciences, KU Leuven, Belgium \\ ${ }^{\mathrm{e}}$ Lab of Sports Medicine, Department of Physical Education and Sport Science, Aristotle University of Thessaloniki, Greece \\ ${ }_{\mathrm{f}}^{\mathrm{f}}$ Information Technologies Institute, Centre for Research and Technology Hellas, Greece \\ ${ }^{\mathrm{g}}$ Insight Centre for Data Analytics, Dublin City University, Ireland \\ ${ }^{\mathrm{h}}$ Health Research Institute, Department of Physical Education and Sport Sciences, University of Limerick, Ireland
}

\section{A R T I C L E I N F O}

\section{Article history:}

Received 30 November 2017

Revised 28 March 2018

Accepted 17 April 2018

\section{Keywords:}

Computerized decision support

Physical activity

Cardiovascular disease

Exercise

Cardiac rehabilitation

\begin{abstract}
A B S T R A C T
Background: Exercise-based rehabilitation plays a key role in improving the health and quality of life of patients with Cardiovascular Disease (CVD). Home-based computer-assisted rehabilitation programs have the potential to facilitate and support physical activity interventions and improve health outcomes.

Objectives: We present the development and evaluation of a computerized Decision Support System (DSS) for unsupervised exercise rehabilitation at home, aiming to show the feasibility and potential of such systems toward maximizing the benefits of rehabilitation programs.

Methods: The development of the DSS was based on rules encapsulating the logic according to which an exercise program can be executed beneficially according to international guidelines and expert knowledge. The DSS considered data from a prescribed exercise program, heart rate from a wristband device, and motion accuracy from a depth camera, and subsequently generated personalized, performance-driven adaptations to the exercise program. Communication interfaces in the form of RESTful web service operations were developed enabling interoperation with other computer systems.

Results: The DSS was deployed in a computer-assisted platform for exercise-based cardiac rehabilitation at home, and it was evaluated in simulation and real-world studies with CVD patients. The simulation study based on data provided from 10 CVD patients performing 45 exercise sessions in total, showed that patients can be trained within or above their beneficial HR zones for $67.1 \pm 22.1 \%$ of the exercise duration in the main phase, when they are guided with the DSS. The real-world study with 3 CVD patients performing 43 exercise sessions through the computer-assisted platform, showed that patients can be trained within or above their beneficial heart rate zones for $87.9 \pm 8.0 \%$ of the exercise duration in the main phase, with DSS guidance.

Conclusions: Computerized decision support systems can guide patients to the beneficial execution of their exercise-based rehabilitation program, and they are feasible.
\end{abstract}

(c) 2018 Elsevier B.V. All rights reserved.

\footnotetext{
* Corresponding author at: Lab of Computing, Medical Informatics and Biomedical Imaging Technologies, School of Medicine, Aristotle University of Thessaloniki, Thessaloniki 54124, Greece.

E-mail address: atriand@auth.gr (A. Triantafyllidis).
}

\section{Introduction}

Substantial evidence suggests that regular physical activity helps to improve the health and well-being of both healthy and chronically ill individuals [1]. Exercise-based rehabilitation in patients with Cardiovascular Disease (CVD) is widely recommended by the medical community in order to reduce their mortality and 
improve their quality of life [2,3]. Today, exercise-based Cardiac Rehabilitation (CR) is mainly performed by patient groups in a controlled environment (e.g., a specialized rehabilitation centre), under the supervision of physiotherapists or cardiac nurses. However, low uptake and poor adherence to exercise-based CR is a major problem which is caused by several barriers, including transport, cost, dislike of group sessions, and self-efficacy issues [4].

A potential solution to the issue of patient adherence to exercise therapy, is the uptake of computerized interventions which can be utilised within the home environment [5]. Computerized interventions enhanced with new and widely available sensing technologies, such as smart watches and depth cameras, can enable physical activity self-tracking on a daily basis without constant supervision by health professionals [6]. In this context, a major challenge is to identify robust ways of assisting patients in unsupervised exercise-based rehabilitation programs to reach their goals, through the processing and evaluation of sensed physical activity data along with potentially available other clinical and behavioural information.

The aim of this paper is to present the design, development and evaluation of a computerized decision support system (DSS), targeting at the personalized and beneficial execution of exercisebased CR programs by CVD patients at their home. The system uses a rule-based approach in order to: a) Process clinical data, as well as data from sensors such as a smart watch and a depth camera, and b) generate actions in terms of adapting the CR program according to the patient's performance. Communication interfaces in the form of web service operations were adopted toward developing an interoperable and extensible system which can be integrated in future home-based exercise platforms. The system has been successfully deployed in PATHway (Physical Activity Towards Health) [7], a computer-assisted platform employing a virtual coach for exercise-based CR at home. Results from the evaluation of the DSS are presented, in terms of performance and guidance of individuals to exercise within or above their beneficial HR zones, as obtained from both simulation and real-world studies.

To the best of our knowledge, this is one of the first studies illustrating the development and evaluation outcomes of a technical infrastructure dedicated for computerized decision support in unsupervised exercise-based CR. Other studies employing rule-based systems have focused on conditions other than CVD. The systems described by Lim et al. [8], for tailored message generation, and Song et al. [9], for autonomous bicycle ergometer training, focus on diabetes and COPD, while Salvi et al. focused on educational and motivational aspects of exercise-based CR [10]. Long-term physical activity outcomes of interventions utilizing computerized decision support have been widely reported $[11,12]$, however it is less known how decision support components should be developed to contribute to the effectiveness of those interventions. To this end, our system is the first-of-its-kind which uniquely processes and evaluates sensed data, e.g., heart rate (HR) and motion accuracy, along with other clinical and behavioural information (e.g., exercise prescription, self-reports on exertion, patient performance history) during unsupervised exercise, toward the beneficial execution of exercise-based rehabilitation programs.

\section{Methodology}

\subsection{Background}

According to international guidelines, an optimal personalized exercise prescription for rehabilitation includes a detailed description of four exercise characteristics, namely Frequency, Intensity, Type, and Time (FITT) [13]. One of the biggest challenges around exercise prescription is making the patients train at the right intensity, i.e., within their beneficial HR zones, which is key for ef- fectiveness [1]. Beneficial HR zones are ideally formulated based on the results of a cardiopulmonary exercise test (CPET). The types of exercises are chosen by the supervisor taking into account basic demographic characteristics of the patients, co-morbidities, and the fitness level of the average participants, as well as the execution during previous sessions. Optimally, the trainer proposes a variety of aerobic and resistance exercises targeting all body parts (legs, arms, trunk, etc.), with different levels of difficulty. Perceived exertion and enjoyment should also be considered when structuring exercise programs [14,15].

\subsection{DSS development}

We followed an iterative approach for developing the DSS, in which engineers and experts in medical informatics (authors AT, DF, AC, DZ, PD, KM, IC, NM) as well as experts in CR, exercise physiology and health and exercise psychology (authors JC, RB, VC, DW, CW) collaborated to identify the functional requirements of the system. In this context, during the initial stage of the development, a review of the clinical guidelines and recommendations [13], was combined with expert knowledge, in order to determine the response of the system according to specific input data (e.g., sensed data, exercise prescription, etc.). The clinical knowledge was coded into deterministic rules in the format of condition-action (IF-THEN), and subsequently the communication interfaces of the system were developed. We chose a rule-based approach mainly because we expected that domain-specific rules would be easily co-designed and understood by experts in CR, exercise physiology and health and exercise psychology, and require limited development effort. Furthermore, rule-based approaches have been used for exercise-based rehabilitation and they have shown their feasibility [16]. To this end, rules were identified by the domain experts toward the safe, beneficial (in terms of patient guidance toward exercising within their optimal HR zones), and personalized (in terms of long-term adaptation of the exercise program) execution of the exercise program.

The DSS development was underpinned by health psychology theory, namely the Behaviour Change Wheel (BCW) [17]. At the core of the BCW, lies a theoretical model describing the catalysts for behaviour, based on "Capability”, i.e., the individual's ability (either physical or psychological) to enact a behaviour, "Opportunity", i.e., the physical (e.g., opportunity afforded by the environment) and social environment (e.g., cultural norms) that enables the behaviour, and "Motivation", i.e., the reflective (e.g., intention and choice) and automatic mechanisms (e.g., habit), that activate or inhibit Behaviour (COM-B) [18]. This model has been used across several studies to aid intervention design and has demonstrated reliability [19]. The BCW has also been used in other studies in the area of decision support for healthcare [20,21]. Further information about the adoption of the BCW and COM-B in PATHway can be found in [22].

\subsection{DSS architecture}

The target was to develop an intelligent middleware which monitors performance and progress of a patient who uses computerized exercise-based $\mathrm{CR}$, and personalizes the weekly exercise program (Fig. 1) [23]. There are three key rule-based components which are working towards these objectives:

(a) Prescreening component: Triggered before the beginning of an exercise session, the prescreening component aims to ensure patient safety. To this end, HR along with blood pressure measurements are obtained through a blood pressure device, and in case of detected abnormal values according to clinical recommendations $[13,24]$, the patient is instructed 


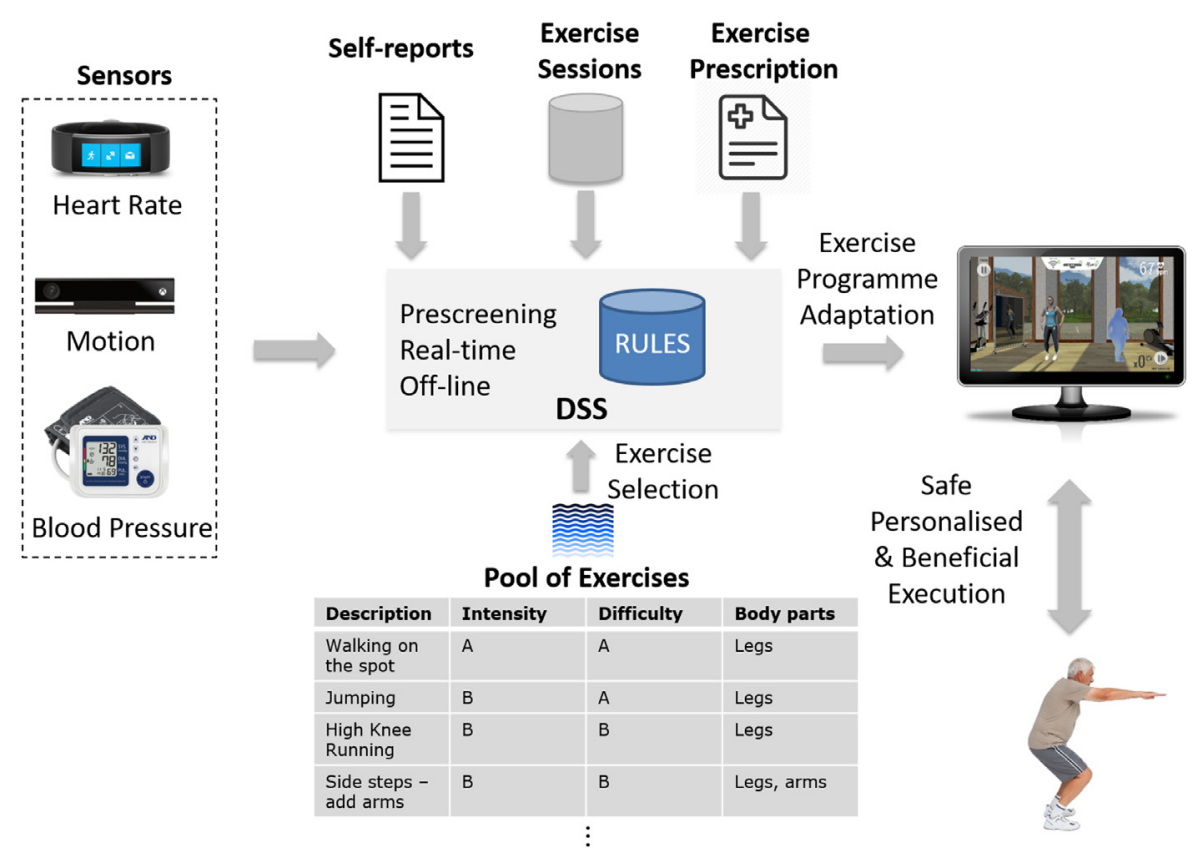

Fig. 1. DSS architecture.

to rest and take the blood pressure again at a later time. When blood pressure readings are high at multiple, consequent readings, the patient is instructed to communicate with a health professional. Self-reports for medication compliance (patient should take his/her prescribed medicines on the day of exercise) and food intake (patient should eat in the last 4 hours before starting exercise), are also obtained to increase the safety while exercising.

(b) Real-time component: Triggered during an exercise session, the real-time component targets at guiding patients toward exercising within their optimal HR zones, according to the patient's exercise prescription (e.g., $150 \mathrm{~min}$ of moderate intensity exercise weekly). The real-time component uses computerized exercise selection to dynamically adjust an exercise session based on a pool of available short-duration (e.g., 1-min) exercises, with different intensities (e.g., highknee running versus walking on the spot), difficulty (in terms of different required levels of balance and coordination), type (aerobic, strength, stretch), and involved body parts (legs, arms, trunk). For example, an exercise of high intensity (e.g., high-knee running) will be dynamically selected in real-time, if the patient is below the beneficial HR zones, while an exercise of low intensity (e.g., walking on the spot) will be selected, if the patient is above the beneficial HR zones.

Additionally, applying motion analysis algorithms in realtime motion capture data acquired with skeleton tracking techniques and depth-sensing devices, results in exercise performance accuracy (motion accuracy) evaluation. The accuracy is estimated and acquired per exercise repetition and cumulatively (repetition set), based on the detection of a priori known physical exercise repetition instances within a sequence of motion data using machine learning techniques (e.g., Adaptive Boosting and Random Forest Regression). Subsequently, the motion data of the detected exercise repetition is being analysed and evaluated, providing numerical and semantic feedback regarding the exercise performance accuracy. Detailed description of the exercise detection algorithms has been given in [25]. The exercise performance evaluation is used in order to evaluate the degree of adherence to the exercise form [26], as well as for motivating the patients toward good execution of the exercise through semantic feedback. In this regard, an exercise of low difficulty will be selected in real-time for execution (e.g., jumping), if the detected cumulative motion accuracy (i.e., the average accuracy of the performed exercise repetitions) during a previous exercise is very low. Finally, the duration of each exercise type and exercised body part is considered ("Time" characteristic in FITT), to ensure variety and balance in exercise selection [1].

(c) Off-line DSS: The off-line DSS is triggered after the completion of an exercise session and targets at long-term adaptions of the exercise program, based on the performance and behaviour of the user ("capability" component of the BCW). As such, the off-line DSS adapts the frequency and duration of required exercise sessions in a week, based on achieved performance according to the exercise prescription goal. Furthermore, the off-line DSS excludes specific exercises from the program when detected motion accuracy is low repeatedly for specific exercises (i.e., user faces difficulty in executing these exercises with the correct form). Based on the acquisition of self-reports for perceived exertion (Borg scale $0-10$ [15]) and enjoyment (scale $0-4$ ) at the end of the exercise session, in case of recurring extremely high exertion or extremely low enjoyment, patients are recommended to perform a different type of physical activity, e.g., outdoors ("opportunity" component of the BCW). Motivational feedback is also triggered for the users when they are close or not to reach their physical activity goals (e.g., on a weekly basis according to their exercise prescription), or when they reach higher goals than the previous week, in order to encourage them accordingly ("motivation" component of the BCW). Finally, if the patient is consistently non-compliant in reaching his/her weekly goals (e.g., 4 weeks in a row), a reduction of $10 \%$ in the weekly goal (in terms of required minutes of physical activity), is initiated, thus assigning a 'graded task' which seems feasible to achieve for participants who may otherwise disengage. 
Table 1

Example rules within the prescreening and off-line DSS components (BP: Blood Pressure, MA: Threshold for acceptable motion accuracy set e.g., to 0.4 in the scale $0-1$ ).

\begin{tabular}{|c|c|c|c|}
\hline & Rule Description & Condition & DSS action \\
\hline : & $\begin{array}{l}\text { Check if the patient had a high } \\
\text { BP/HR AND answer to question } \\
\text { about today's medication } \\
\text { compliance is NO more than once } \\
\text { during last } 3 \text { sessions within } 14 \text { days }\end{array}$ & $\begin{array}{l}\text { If answer to question about taking medication, is NO AND } \\
\text { answer to question about medication is } \mathrm{NO} \text { for }>=2 \text { times } \\
\text { during last } 14 \text { days AND systolic } \mathrm{BP}>=180 \text { OR resting HR } \\
>=75 \% \text { peak HR AND (systolic } \mathrm{BP}>=180 \text { OR resting HR } \\
>=75 \% \text { peak HR) has been triggered }>=2 \text { times during last } \\
14 \text { days }\end{array}$ & $\begin{array}{l}\text { Instruct patient not to } \\
\text { start exercise }\end{array}$ \\
\hline \multirow{3}{*}{ 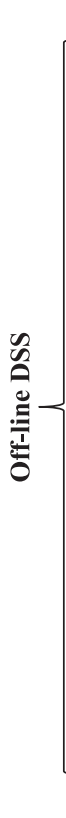 } & Consider session acceptable & $\begin{array}{l}\text { If total patient mean accuracy during session }>=\text { MA AND } \\
\text { exercise session duration }>50 \% \text { of expected session duration } \\
\text { AND time spent within or above beneficial HR zones during } \\
\text { main phase }>50 \% \text { of total main phase duration }\end{array}$ & $\begin{array}{l}\text { Assign this exercise } \\
\text { session as acceptable } \\
\text { (otherwise the session } \\
\text { is assigned as } \\
\text { unacceptable and the } \\
\text { patient must re } \\
\text { perform the exercise } \\
\text { session) }\end{array}$ \\
\hline & $\begin{array}{l}\text { Exclude specific exercise when } \\
\text { accuracy is low for more than } 3 \\
\text { consecutive appearances of the } \\
\text { exercise }\end{array}$ & $\begin{array}{l}\text { If exercise consecutive appearances }>=3 \text { AND mean } \\
\text { accuracy }<\mathrm{MA} \text { for the sessions }\end{array}$ & $\begin{array}{l}\text { Assign this exercise } \\
\text { id as excluded for all } \\
\text { the next exercise } \\
\text { sessions for this } \\
\text { patient for } 3 \text { weeks. }\end{array}$ \\
\hline & $\begin{array}{l}\text { Motivate patient when he/she is on } \\
\text { track to meet the weekly goal near } \\
\text { week's end }\end{array}$ & $\begin{array}{l}\text { If (time is end of day } 5 \text { of the weekly exercise } \\
\text { program) AND SUM (session_durations) }>50 \% \\
\text { of weekly physical activity goal }\end{array}$ & $\begin{array}{l}\text { Trigger message: } \\
\text { "You have nearly } \\
\text { reached your } \\
\text { physical activity } \\
\text { goal for this week. } \\
\text { There are just two } \\
\text { days left! You can } \\
\text { do it!" }\end{array}$ \\
\hline
\end{tabular}

Table 2

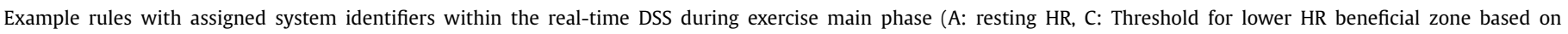

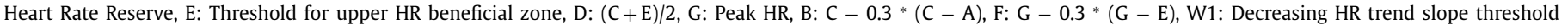
$\left(-30^{\circ}\right)$, W2: Increasing HR trend slope threshold $\left.\left(+30^{\circ}\right)\right)$.

\begin{tabular}{|c|c|c|c|c|}
\hline \multicolumn{3}{|c|}{ Rule conditions } & \multicolumn{2}{|c|}{ Actions (for next exercise) } \\
\hline ID & Accuracy & Heart Rate & Intensity & Difficulty \\
\hline$\# 1$ & Medium/high & $\begin{array}{l}\text { (HRmean }<\text { C AND HRmean }>\text { B AND slope }>\text { W2) OR }(\text { HRmean }>\text { C AND } \\
\text { HRmean }<\text { D AND slope }>\text { W2) OR (HRmean }>\text { D AND HRmean }<\text { E AND } \\
\text { slope }<\text { W1) OR }(\text { HRmean }>\text { E AND HRmean }<\text { F AND slope }<\text { W1) }\end{array}$ & - & $\uparrow /-$ \\
\hline \#2 & Medium/high & $\begin{array}{l}(\text { HRmean }<\text { B) OR }(\text { HRmean }>\text { B AND HRmean }<\text { C AND slope }<\text { W } 1) \text { OR } \\
(\text { HRmean }>\text { C AND HRmean }<\text { D AND slope }<\text { W1 })\end{array}$ & $\uparrow$ & - \\
\hline \#3 & Medium/high & $\begin{array}{l}\text { (HRmean }>\text { D AND HRmean }<\text { E AND slope }>\text { W2) OR }(\text { HRmean }>\text { E AND } \\
\text { HRmean }<\text { F AND slope }>\text { W2) OR }(\text { HRmean }>\text { F) }\end{array}$ & $\downarrow$ & - \\
\hline$\# 4$ & Low & (HRmean $>$ E AND slope $>$ W2) OR HRmean $>$ F & $\downarrow$ & $\downarrow$ \\
\hline \#5 & Low & (HRmean $<$ F AND HRmean $>$ E) AND slope $<$ W2 & - & $\downarrow$ \\
\hline \#6 & Low & (HRmean $<$ C AND slope $<$ W2) OR HRmean $>$ B & $\uparrow$ & $\downarrow$ \\
\hline \#7 & Low & (HRmean $>$ B AND HRmean $<$ C) AND slope $>$ W1 & - & $\downarrow$ \\
\hline \#8 & \multicolumn{2}{|c|}{ If none of rules $1-7$ applies } & - & - \\
\hline
\end{tabular}

-: Same, $\uparrow$ : Increase, $\downarrow$ : Decrease

\subsection{Technical infrastructure for exercise program adaptation}

All rules in the DSS were coded as condition-action in the format IF-THEN in the Python programming language, and they were assigned an identifier. Python was selected because of the useful characteristics it provides, such as dynamic and efficient memory management, cross-platform availability, and excellent code readability, all contributing to rapid prototyping. Examples of rules for the prescreening and off-line DSS components are given in Table 1 , and for the real-time component in Table 2. Specific zones were considered for motion accuracy (low, medium, high) for each exercise according to thresholds set by experts in the scale $0-1$ (e.g. $<0.4$ can be low accuracy based on the virtual coach reference accuracy), and for HR as a percentage of the Heart Rate Reserve (calculated as: Peak HR - resting HR) based on the CPET. Thresholds for the least mean squares linear fit slope were used to denote a decreasing or increasing trend of the HR signal [27,28].

We adopted a service-oriented architecture, in which DSS communication interfaces were developed as RESTful web service operations, which can enable the interoperability and easy extension 


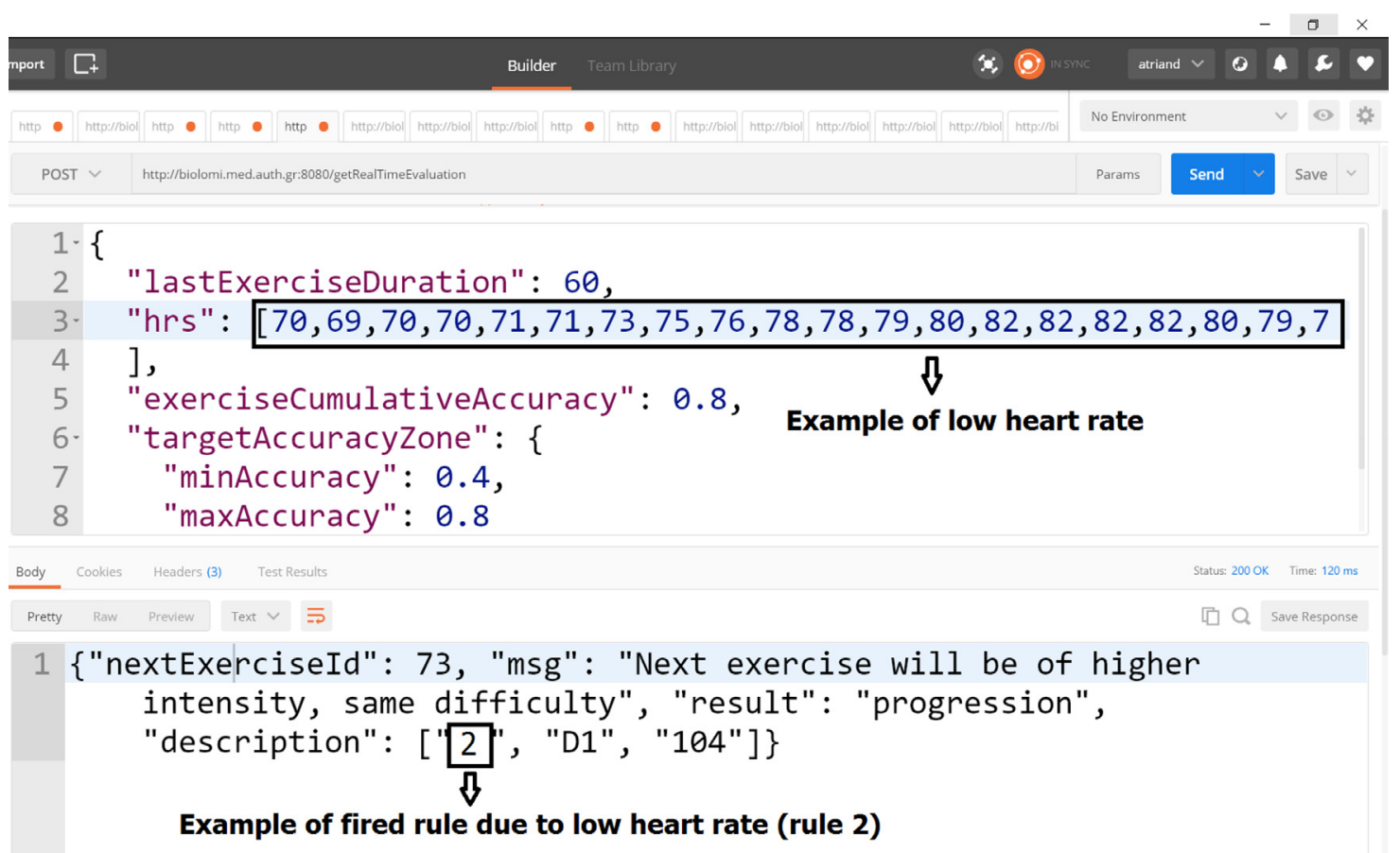

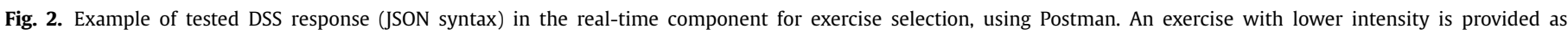
output due to user's high heart rate according to rule 2 .

of communication systems [29]. In this direction, web service operations linked to the prescreening, real-time, and off-line components of the DSS (e.g., getPreScreeningEvaluation(), getRealTimeEvaluation(), getAfterSessionExcludedExercises(), etc.) were developed, enabling their call by client applications or systems. The JSON format $^{1}$ was used to represent acquired input data (rule conditions) and generated response data (rule actions) of the DSS, mainly because it is lightweight for data interchange and easy to parse. We used the Postman tool, ${ }^{2}$ an easy-to-use testing environment for web interfaces, in order to test the response of all DSS web service operations (Fig. 2), and fix any input/output errors during the development process, prior to their deployment in the system's production server. The DSS runs in a Python 2.7 WSGI capable server and requires $16 \mathrm{~GB}$ of memory as well as a 64 -bit Windows $8 / 10$ or Linux environment to ensure its smooth operation. System usage logs were implemented and used to capture the fired rules during user interaction with the system, enabling the automatic collection of all DSS responses for exercise program adaptation. The development of the RESTful communication interfaces enabled the integration of the DSS in the PATHway platform ${ }^{3}$ (Fig. 3), in which communication was required with the system client (front-end) application to deliver the adaptations of the CR program, e.g., dynamic exercise selection in real-time. In PATHway, the Microsoft Xbox One Kinect sensor [30] (as depth-sensing device) and the Microsoft Band HR tracking device [31] (as smart watch) were used to meet requirements for valid motion capture and HR monitoring. Since we adopted a RESTful interoperable architecture, the selection of specific sensing devices does not affect the DSS operation, and therefore other devices can also be integrated in the future.

\footnotetext{
1 JSON: https://www.json.org/, last accessed on 22nd March 2018.

2 PostMan Tool: https://www.getpostman.com/, last accessed on 9th October 2017.

3 Demonstration of the PATHway system: http://pathway2health.eu/demos/, last accessed on 9th October 2017.
}

2.5. Methodology for system evaluation in simulation and real-world studies

In the context of system evaluation, we firstly performed a simulation study to explore the feasibility of the system and the usefulness of the adopted rule-based approach, in terms of guiding CVD patients to exercise within their beneficial HR zones. To this end, we recruited CVD patients participating in a community exercise-based CR program (Thessaloniki, Greece), in order to capture their HR response during exercise, according to the instructions of a trainer. HR was tracked via a wristband device, Scosche Rhythm (Scosche Industries, Oxnard, California, USA), which has been found to be accurate [31]. A Kinect sensor was also used to capture the exercise execution and to annotate the exercises (i.e., their intensity and difficulty) retrospectively according to trainers' expertise. To this end, individual exercises were manually tagged with a low (A) or high (B) intensity label after watching the Kinect recordings. Ethical approval for the conduction of the study was granted by the research committee of the Aristotle University of Thessaloniki, Greece (Prot. No.: 39768/2016). After completing data collection and annotation, we followed a linear modelling approach based on the HR response for exercises of intensity A and B, reported in our previous work [28], to determine the percentage of time spent within or above the beneficial HR zones when the rules of the real-time component (Table 2) are utilized.

We further explored the use and initial outcomes of computerized exercise selection in the real-time component of the DSS, in an ethically approved clinical trial with CVD patients performing unsupervised exercise-based CR at home through the PATHway system. The protocol of the clinical trial is reported in [7]. The results of the exercise response from participants in terms of heart rate and motion accuracy, as well as the fired rules, were acquired from the system usage logs from August to October 2017. The systematic assessment of the off-line DSS according to its capabilities in the long-term decision horizon described above, will be presented after the completion of the trial. 


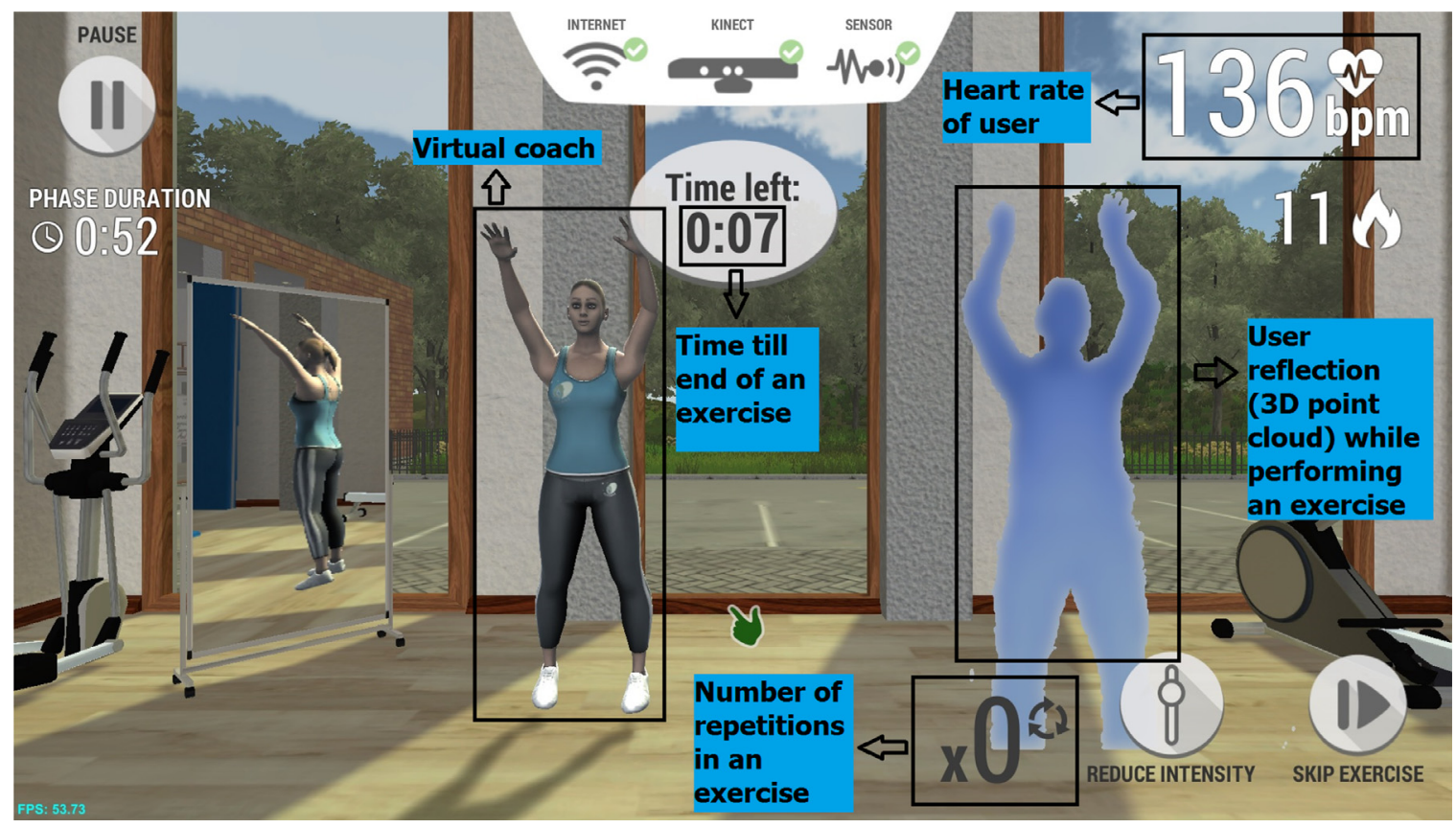

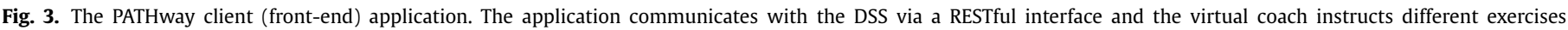
according to DSS response (real-time component), in order to guide patients to exercise within their beneficial heart rate zones.

\section{Results}

\subsection{Technical evaluation}

The technical evaluation of the system focused on the performance of the DSS web service operations in terms of measuring their response time. We used the JMeter tool $^{4}$ to perform concurrent testing with 10,20 , and 100 users for 10 tests each, using a ramp-up period of $1 \mathrm{~s}$, for which the most-used operation of our system, i.e., the real-time selection of exercises which is triggered every minute (getRealTimeEvaluation()) had a max response time of 164, 444, and 2842 milliseconds respectively on average. Other DSS operations linked to the pre-screening component (getPrescreeningEvaluationResult()) and the off-line DSS (e.g., getAfterSessionExcludedExercises()), had max response times of 29, 31, and 331 milliseconds, and 43, 100, and 2244 milliseconds on average respectively, yielding also acceptable results.

\subsection{Simulation study}

The study was based on the exercise response of 10 (6 male, 4 female) CVD patients ( $70.3 \pm 6.5$ years). In total, 45 half-hour exercise sessions were monitored, in which a trainer instructed different short-duration exercises $(30.1 \pm 11.5$ seconds for low intensity, $30.2 \pm 10.5$ seconds for high intensity exercises). CPET results were obtained from all participating patients (mean resting HR: $69 \pm 12$, mean peak HR: $129 \pm 18$ ) in order to specify the beneficial HR zones according to the Karvonen's formula [13]. Based on trainers' feedback, the intensity of the program was moderate, while monitored patients did not face any difficulty in executing the instructed exercises. As such, a medium/high motion accuracy was assigned for all monitored participants when performing their exercise session.

We modelled the HR response during exercise at an intensity level of $A$ and $B$, expressing the linear change of $H R$ based on the starting HR in each exercise and its intensity (R-

\footnotetext{
${ }^{4}$ JMeter Tool: http://jmeter.apache.org/, last accessed on 9th October 2017.
}

squared $=0.24 \pm 0.14$; Root Mean Square Error $($ RMSE $)=4.5 \pm 1.5$ and R-squared $=0.25 \pm 0.17$; RMSE $=4.7 \pm 2.5$, for linear models of intensity A and B exercises, respectively) (Fig. 4a). Then, we applied the Monte Carlo technique [32] for $10^{3}$ repetitions to explore how the model responds to randomly-generated inputs (Fig. 4b). Finally, we computed the average percentage of time CVD patients were exercising within or above their beneficial HR zones, based on the simulated HR data with three options: Option 1: application of the DSS rules, option 2: alternation of the exercises during the main phase in a 2:1 pattern (two exercises of intensity $B$ followed by one exercise of intensity A, option 3: random sequence of exercises (Table 3 ).

The average percentage of time CVD patients were exercising within or above their beneficial HR zones in the community rehabilitation programs supervised by a trainer (real-life sessions) was equal to $48.9 \pm 31.5 \%$ of the exercise main phase duration (i.e., excluding the duration of warm-up and cool-down phases). Based on the results we obtained after applying the simulation technique described above, CVD patients can be trained within or above their beneficial HR zones for $67.1 \pm 22.1 \%$ of the exercise duration in the main phase, when they are guided with the DSS rules during real-time. In Fig. 5, the participants' percentage of the exercise main phase duration their heart rate remained within or above the beneficial zones in real-life sessions and simulated sessions after applying the DSS rules and the Monte Carlo technique, can be seen. The simulation performed particularly well in those patients whose performance in real-life sessions was low (participants 2, 6 and 7 ). In participant 4 , the simulation yielded similar results with the real-life sessions, possibly because of a high RMSE detected in the linear models of low and high intensity for this particular participant.

\subsection{Exercise sessions by CVD patients interacting with PATHway}

In total, we were able to analyse 43 exercise sessions of at least 30 min duration (sessions were done on different days by each participant) by 3 CVD patients ( 1 male, 2 female), in which the median perceived exertion in the Borg Scale (0: nothing at all, 10: 


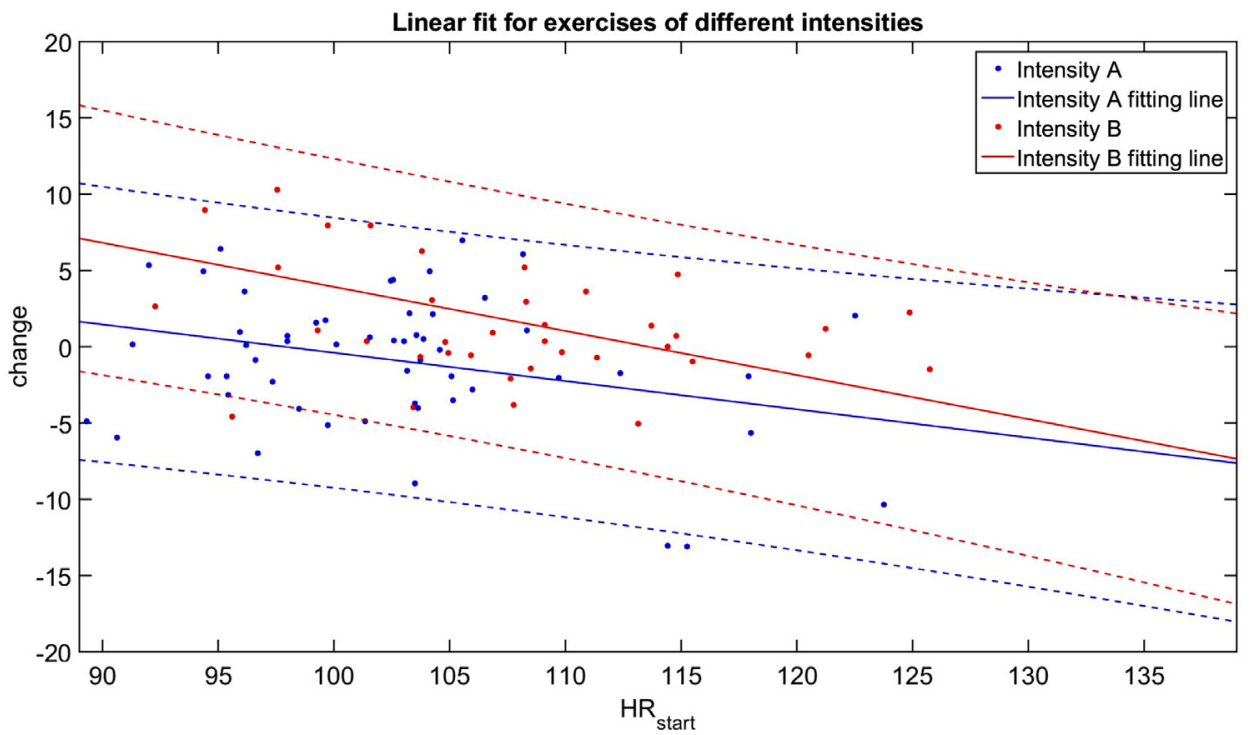

(a)

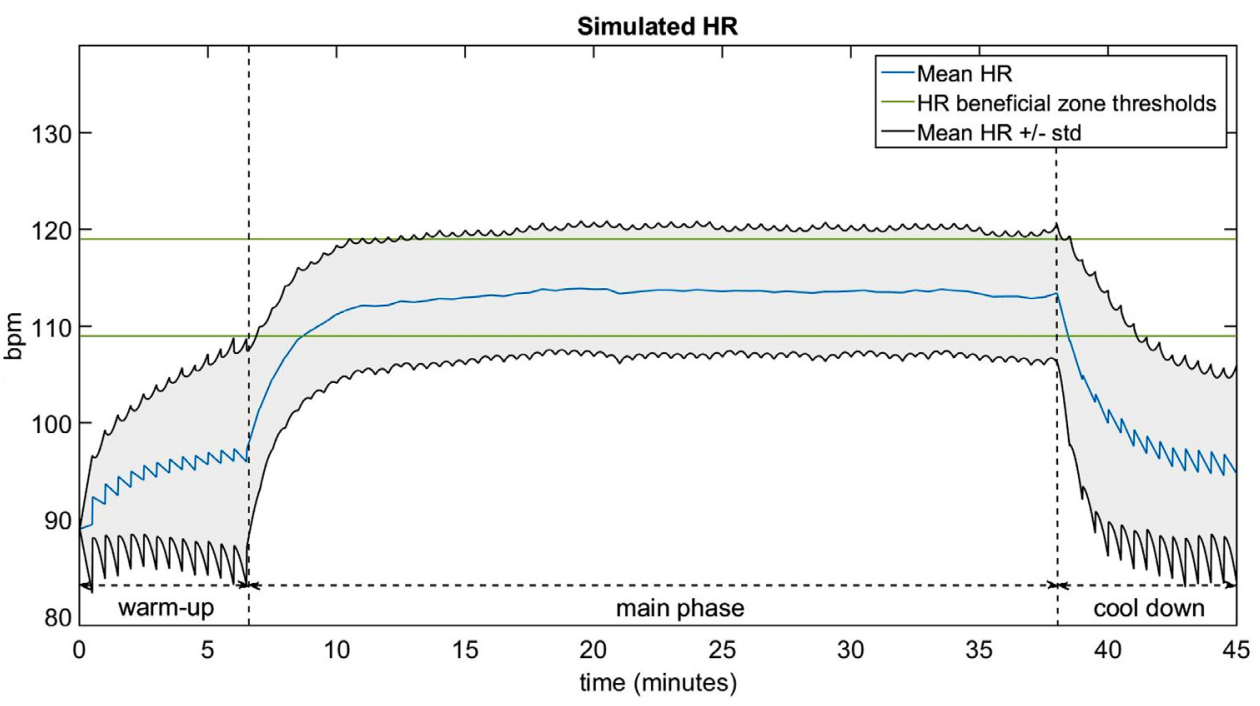

(b)

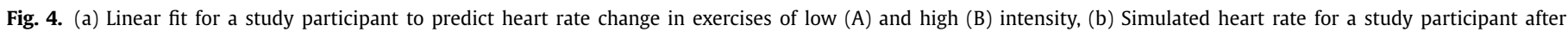
applying the DSS rules and Monte-Carlo.

Table 3

Time spent within or above beneficial HR zones (\% of the main phase duration).

\begin{tabular}{|c|c|c|c|c|}
\hline & Option 1 (rules) & Option 2 (2:1 pattern) & Option 3 (random) & Real-life sessions \\
\hline Percentage of time within or above beneficial HR zones (\%) & $67.1 \pm 22.1$ & $61.4 \pm 26.9$ & $56.6 \pm 28.7$ & $48.9 \pm 31.5$ \\
\hline
\end{tabular}

maximal) was equal to 4 (somewhat strong), and median enjoyment in a $0-4$ scale ( 0 : not at all enjoyable, 4 : very enjoyable) was equal to 2 (quite enjoyable). The participants had a median resting HR of 54 beats per minute (range 45-55) and median peak HR of 164 beats per minute (range 108-179) based on CPET results (Table 4).

Patients achieved to be within or above their beneficial heart rate zones for $87.9 \pm 8.0 \%$ of the duration of the exercise main phase on average, which is considerably better than the threshold of $50 \%$ we have set in our rules for acceptable exercise sessions in terms of HR response (Table 1, row 2). Most fired rules were rule \#1 (572 times) to increase/maintain difficulty and rule \#3 (410 times) to lower intensity, while less fired rules were those for decreasing difficulty (72 times), and rule \#2 for progressing to an exercise with higher intensity (21 times), which shows that patients had high accuracy in performing their exercises and they were constantly achieving to be within or above their beneficial HR zones. Interestingly, participant 1 seemed to be constantly above the beneficial HR zones, and as a result rule \#3 was fired in 395 occasions.

In order to measure the effectiveness of the DSS in patient guidance, we introduced two metrics: a) Recovery from low $H R$ events, which measures the percentage of occasions (number of exercises) in which the DSS guides the patient to exercise within the beneficial HR zones immediately after a low HR event, i.e., when the average HR is below the beneficial HR zones, and b) Recovery 


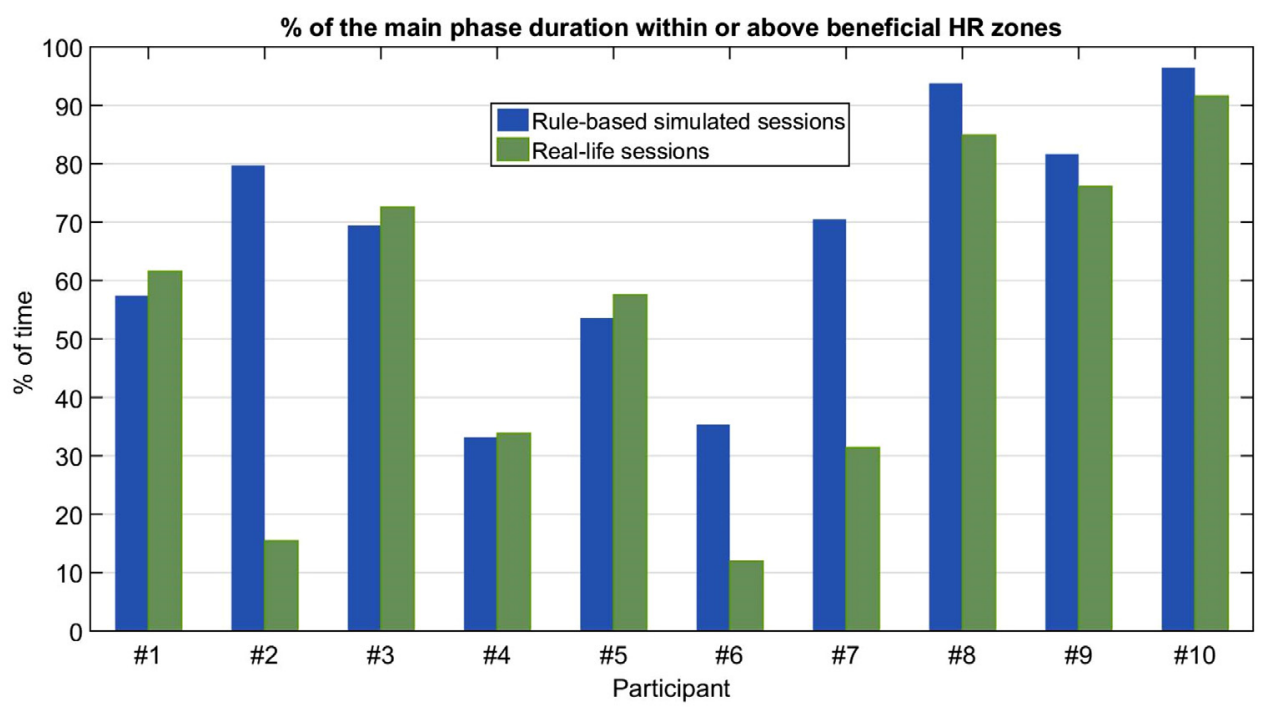

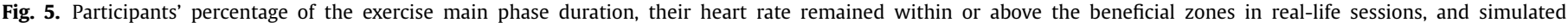
sessions after applying the DSS rules and the Monte Carlo technique.

Table 4

CVD patients' performance in unsupervised exercise during interaction with PATHway.

\begin{tabular}{|c|c|c|c|c|c|}
\hline Participant & $\begin{array}{l}\text { Resting HR (beats } \\
\text { per minute) }\end{array}$ & $\begin{array}{l}\text { Peak HR (beats per } \\
\text { minute) }\end{array}$ & $\begin{array}{l}\text { Number of } \\
\text { monitored exercise } \\
\text { sessions }\end{array}$ & $\begin{array}{l}\text { Average session } \\
\text { duration (minutes) }\end{array}$ & $\begin{array}{l}\text { Average \% time of } \\
\text { main phase } \\
\text { duration that } \\
\text { participant is } \\
\text { within or above } \\
\text { beneficial HR zones }\end{array}$ \\
\hline 1 & 45 & 108 & 21 & 39 (range $31-64$ ) & $99.2 \pm 1.1$ \\
\hline 2 & 55 & 164 & 17 & 32 (range $31-35$ ) & $82.5 \pm 14.5$ \\
\hline 3 & 54 & 179 & 5 & 31 (range $31-32$ ) & $81.9 \pm 9.0$ \\
\hline
\end{tabular}

Table 5

Recovery from low HR and low motion accuracy events.

\begin{tabular}{|c|c|c|c|c|c|c|c|}
\hline Participant & $\begin{array}{l}\text { Low HR events } \\
\text { (Number of } \\
\text { exercises that } \\
\text { average HR is } \\
\text { below beneficial } \\
\text { HR zones) }\end{array}$ & $\begin{array}{l}\text { Recovery from low } \\
\text { HR event }-1 \text { min } \\
\text { time window }\end{array}$ & $\begin{array}{l}\text { Recovery from low } \\
\text { HR event }-2 \text { min } \\
\text { time window }\end{array}$ & $\begin{array}{l}\text { Recovery from low } \\
\text { HR event }-3 \text { min } \\
\text { time window }\end{array}$ & $\begin{array}{l}\text { Low motion } \\
\text { accuracy events } \\
\text { (Number of } \\
\text { exercises that } \\
\text { average motion } \\
\text { accuracy is below } \\
\text { set threshold) }\end{array}$ & $\begin{array}{l}\text { Recovery from low } \\
\text { motion accuracy } \\
\text { event - } 1 \text { min time } \\
\text { window }\end{array}$ & $\begin{array}{l}\text { Recovery from low } \\
\text { motion accuracy } \\
\text { event }-2 \text { min time } \\
\text { window }\end{array}$ \\
\hline 1 & $2(100 \%)$ & $2(100 \%)$ & - & - & $50(100 \%)$ & $44(88 \%)$ & $50(100 \%)$ \\
\hline 2 & $55(100 \%)$ & $25(46 \%)$ & 47 (86\%) & $52(95 \%)$ & $37(100 \%)$ & $29(79 \%)$ & $37(100 \%)$ \\
\hline 3 & $20(100 \%)$ & $10(50 \%)$ & $16(80 \%)$ & $18(90 \%)$ & $8(100 \%)$ & $7(88 \%)$ & $8(100 \%)$ \\
\hline \multicolumn{2}{|c|}{ Average recovery percentage } & $65 \%$ & $83 \%$ & $93 \%$ & & $85 \%$ & $100 \%$ \\
\hline
\end{tabular}

from low motion accuracy events, which measures the percentage of occasions in which the DSS guides the patient to exercise in a good form (as indicated by motion accuracy thresholds set by health professionals for every exercise), immediately after a low motion accuracy event. As illustrated in Table 5, the recovery from low HR events in a 2-min and 3-min window reached to $83 \%$ and $93 \%$ respectively, while the recovery from low motion accuracy events in a 1 -min and 2 -min time window reached to $85 \%$ and $100 \%$ respectively, which shows the value of the DSS in unsupervised exercise guidance.

\section{Discussion}

Patients' adherence to exercise therapy is considered to be a key factor in improving their health and well-being. Computerassisted physical activity interventions deployed at home or other environments $[8,33]$ have been found to facilitate patient engage- ment with regular physical activity and bring benefits in everyday patient monitoring and coaching. The capability to use such interventions conveniently, anytime and without supervision, adds significant value to their usefulness and facilitates patient independent living [34]. In this direction, computer systems for exercisebased rehabilitation are required to be smart, personalized, robust and adaptive, to correspond to changing patient requirements and bring expected health outcomes.

DSSs have traditionally focused on assisting health professionals in clinical decision making $[35,36]$. The shift of focus to the patient [37], in conjunction with the advent of pervasive computing technology [38] - mobile devices, smart sensors, smart watches, etc. has enabled the development of DSSs for patients $[39,40]$, which can be used to assist them in daily self-management of their condition. The current work contributed in the development of such systems by presenting a rule-based DSS for patient guidance in unsupervised exercise-based rehabilitation. 
A multi-component, interoperable and performance-driven DSS toward enhancing computerized exercise-based rehabilitation programs at home, was presented. The development framework of our system, e.g., formulation of simple rules, communication interfaces to interoperate with other systems, and system usage logs to capture the way the system is adapted, was shown, to increase the understanding about the development of robust DSSs. An additional advantage of the proposed system is that this is not tightly coupled to specific sensing devices, and therefore it can be sustainable as technology evolves. Besides presenting the generic capabilities of the system, we focused on achieving a beneficial response during exercise through dynamic program adaptations. In this context, both simulation and real-world studies were conducted to evaluate the DSS. The simulation study provided a proof-of-concept for the usefulness of the DSS rules in guiding patients to exercise within their beneficial heart rate zones. The real-world pilot study with CVD patients provided evidence on the effectiveness of our rulebased approach for unsupervised exercise-based CR, by showing the use and outcomes of computerized exercise selection based on user's heart rate and motion accuracy.

Our work is limited in terms of the number of subjects participating in the described studies and their duration. Therefore, longitudinal studies with a larger number of participants are needed to further explore the effectiveness of our system especially on the long-term decision horizon. Given that sufficient amount of data is collected in such studies, specific personal models of exercise which benefit specific individuals the most can be identified. Machine learning algorithms can be applied to predict patient performance on the short-term (within an exercise session) and outcomes on the long-term (e.g., improvement of cardiovascular fitness as identified in CPET results), and feed their results to the DSS operation by enabling the formulation of new rules. Upon the availability of high heart rate events by several patients, i.e., occasions in which the heart rate trends toward reaching the peak heart rate, we would be able to examine whether the DSS rules contribute in patient safety. Finally, the association of motivational messages and behavioural information such as exertion and enjoyment, with patient adherence and clinical outcomes in the longterm, will facilitate additional insights on the usefulness of DSSs in personalized exercise-based rehabilitation.

In conclusion, the aim of this paper was to present a computerized system capable of collecting, processing and evaluating diversified data, and generating personalized, performancedriven adaptations to unsupervised exercise-based rehabilitation programs, which can be shared with other systems or components. Experimental results showed the effectiveness of the system in beneficial home-based exercise therapy. The operation of such systems and their real-life evaluation can contribute in increasing our understanding on the way optimal benefits from the use of physical activity interventions can be achieved. In this direction, researchers and designers of computer-assisted exercise-based rehabilitation systems can leverage the described approach, in order to achieve improved rehabilitation outcomes.

\section{Summary points}

\section{What was already known on the topic?}

- Exercise-based cardiac rehabilitation improves health and quality of life. Computer-assisted rehabilitation programs delivered at home have the potential to increase effectiveness of as well as adherence to regular exercise.

What this study added to our knowledge?

- To the best of our knowledge, there has been no systematic approach to the design, development and evaluation of dedicated computerized systems in tailoring exercise-based cardiac rehabilitation programs for the home environment.

- A computerized decision support system was developed, adopting a rule-based approach to evaluate sensed data such as heart rate and motion accuracy, along with other clinical and behavioural information, in order to deliver a safe, personalized and beneficial execution of an exercise program.

- Simulation and real-world experimental studies with patients with cardiovascular disease showed the feasibility and effectiveness of the computerized decision support system.

- Computerized decision support systems can guide patients to the beneficial execution of their exercise-based rehabilitation program, and they are feasible.

\section{Authors' contributions}

Authors IC and NM were responsible for the study conception; Authors AT and DF led the design, development and evaluation of the described system and received contributions from all authors; AT wrote a first draft of the manuscript and all other authors contributed to the final version. All authors have read and agreed to the paper being submitted as it is.

\section{Acknowledgements}

The work described in this paper was supported by the European Union's Horizon 2020 Framework Program for Research and Innovation Action under Grant Agreement no. 643491, 'PATHway: Technology enabled behavioural change as a pathway towards better self-management of CVD'.

\section{Conflicts of interest}

The authors of this manuscript declare no conflicts of interest.

\section{References}

[1] L. Vanhees, N. Geladas, D. Hansen, E. Kouidi, J. Niebauer, Z. Reiner, V. Cornelissen, S. Adamopoulos, E. Prescott, M. Börjesson, B. Bjarnason-Wehrens, H.H. Björnstad, A. Cohen-Solal, V. Conraads, D. Corrado, J. De Sutter, P. Doherty, F. Doyle, D. Dugmore, Ø. Ellingsen, R. Fagard, F. Giada, S. Gielen, A. Hager, M. Halle, H. Heidbüchel, A. Jegier, S. Mazic, H. McGee, K.P. Mellwig, M. Mendes, A. Mezzani, N. Pattyn, A. Pelliccia, M. Piepoli, B. Rauch, A. Schmidt-Trucksäss, T. Takken, F. van Buuren, D. Vanuzzo, Importance of characteristics and modalities of physical activity and exercise in the management of cardiovascular health in individuals with cardiovascular risk factors: recommendations from the EACPR. Part II., Eur. J. Prev. Cardiol. 19 (2012) 1005-1033, doi:10.1177| 1741826711430926.

[2] V.A. Sagar, E.J. Davies, S. Briscoe, A.J.S. Coats, H.M. Dalal, F. Lough, K. Rees, S. Singh, R.S. Taylor, Exercise-based rehabilitation for heart failure: systematic review and meta-analysis, Open Heart 2 (2015) e000163, doi:10.1136/ openhrt-2014-000163.

[3] B.S. Heran, J.M. Chen, S. Ebrahim, T. Moxham, N. Oldridge, K. Rees, D.R. Thompson, R.S. Taylor, Exercise-based cardiac rehabilitation for coronary heart disease, Cochrane Database Syst. Rev. (2011) CD001800, doi:10.1002/14651858. CD001800.pub2.

[4] J. Daly, A.P. Sindone, D.R. Thompson, K. Hancock, E. Chang, P. Davidson, Barriers to participation in and adherence to cardiac rehabilitation programs: a critical literature review, Prog. Cardiovasc. Nurs. 17 (2002) 8-17, doi:10.1111/j. 0889-7204.2002.00614.x.

[5] I.-L. Aamot, S.H. Forbord, K. Gustad, V. Løckra, A. Stensen, A.T. Berg, H. Dalen, T. Karlsen, A. Støylen, Home-based versus hospital-based high-intensity interval training in cardiac rehabilitation: a randomized study, Eur. J. Prev. Cardiol. 21 (2014) 1070-1078, doi:10.1177/2047487313488299.

[6] J.C. Rawstorn, N. Gant, A. Direito, C. Beckmann, R. Maddison, Telehealth exercise-based cardiac rehabilitation: a systematic review and meta-analysis, Heart 102 (2016) 1183-1192, doi:10.1136/heartjnl-2015-308966.

[7] J. Claes, R. Buys, C. Woods, A. Briggs, C. Geue, M. Aitken, N. Moyna, K. Moran, N. McCaffrey, I. Chouvarda, D. Walsh, W. Budts, D. Filos, A. Triantafyllidis, N. Maglaveras, V.A. Cornelissen, PATHway I: design and rationale for the investigation of the feasibility, clinical effectiveness and cost-effectiveness of a technology-enabled cardiac rehabilitation platform, BMJ Open 7 (2017), doi:10. 1136/bmjopen-2017-016781

[8] S. Lim, S.M. Kang, K.M. Kim, J.H. Moon, S.H. Choi, H. Hwang, H.S. Jung, K.S. Park, J.O. Ryu, H.C. Jang, Multifactorial intervention in diabetes care using 
real-time monitoring and tailored feedback in type 2 diabetes, Acta Diabetol. 53 (2016) 189-198, doi:10.1007/s00592-015-0754-8.

[9] B. Song, M. Becker, M. Gietzelt, R. Haux, M. Kohlmann, M. Schulze, U. Tegtbur, K.-H. Wolf, M. Marschollek, Feasibility study of a sensor-based autonomous load control exercise training system for COPD patients, J. Med. Syst. 39 (2015) 150, doi:10.1007/s10916-014-0150-x.

[10] D. Salvi, M. Ottaviano, S. Muuraiskangas, A. Martínez-Romero, C. Vera-Muñoz, A. Triantafyllidis, M.F. Cabrera Umpiérrez, M.T. Arredondo Waldmeyer, E. Skobel, C. Knackstedt, H. Liedes, A. Honka, J. Luprano, J.G. Cleland, W. Stut, C. Deighan, An m-Health system for education and motivation in cardiac rehabilitation: the experience of HeartCycle guided exercise, J. Telemed. Telecare (2017), doi:10.1177/1357633X17697501.

[11] R.D. Reid, L.I. Morrin, L.J. Beaton, S. Papadakis, J. Kocourek, L. McDonnell, M.E. Slovinec D'Angelo, H. Tulloch, N. Suskin, K. Unsworth, C. Blanchard, A.L. Pipe, Randomized trial of an internet-based computer-tailored expert system for physical activity in patients with heart disease, Eur. J. Prev. Cardiol. 19 (2012) 1357-1364, doi:10.1177/1741826711422988.

[12] M. Varnfield, M. Karunanithi, C.-K. Lee, E. Honeyman, D. Arnold, H. Ding, C. Smith, D.L. Walters, Smartphone-based home care model improved use of cardiac rehabilitation in postmyocardial infarction patients: results from a randomised controlled trial, Heart 100 (2014) 1770-1779, doi:10.1136/ heartjnl-2014-305783.

[13] P.D. Thompson, R. Arena, D. Riebe, L.S. Pescatello, ACSM's new preparticipation health screening recommendations from ACSM's guidelines for exercise testing and prescription, ninth edition, Curr. Sports Med. Rep. 12 (2013) 215-217, doi:10.1249/JSR.0b013e31829a68cf.

[14] T.D. Raedeke, The relationship between enjoyment and affective responses to exercise, J. Appl. Sport Psychol. 19 (2007) 105-115, doi:10.1080/ 10413200601113638.

[15] G. Borg, P. Hassmén, M. Lagerström, Perceived exertion related to heart rate and blood lactate during arm and leg exercise, Eur. J. Appl. Physiol. Occup. Physiol. 56 (1987) 679-685, doi:10.1007/BF00424810.

[16] W. Zhao, D.D. Espy, M.A. Reinthal, H. Feng, A feasibility study of using a single Kinect sensor for rehabilitation exercises monitoring: a rule based approach, in: 2014 IEEE Symp. Comput. Intell. Healthc. E-Health, IEEE, 2014, pp. 1-8, doi:10.1109/CICARE.2014.7007827.

[17] S. Michie, L. Atkins, R. West, The behaviour change wheel: a guide to designing interventions, Off. Mag. Can. Soc. Physician Leaders. 2 (2015) 14-17.

[18] F. Barker, L. Atkins, S. de Lusignan, Applying the COM-B behaviour model and behaviour change wheel to develop an intervention to improve hearing-aid use in adult auditory rehabilitation, Int. J. Audiol. 55 (2016) S90-S98, doi:10.3109/ 14992027.2015 .1120894$.

[19] S. Michie, M.M. van Stralen, R. West, The behaviour change wheel: a new method for characterising and designing behaviour change interventions, Implement. Sci. 6 (2011) 42, doi:10.1186/1748-5908-6-42.

[20] M.A. Maar, K. Yeates, Z. Toth, M. Barron, L. Boesch, D. Hua-Stewart, P. Liu, N. Perkins, J. Sleeth, M.J. Wabano, P. Williamson, S.W. Tobe, Unpacking the black box: a formative research approach to the development of theory-driven, evidence-based, and culturally safe text messages in mobile health interventions, JMIR mHealth uHealth 4 (2016) e10, doi:10.2196/mhealth.4994.

[21] D. Praveen, A. Patel, A. Raghu, G.D. Clifford, P.K. Maulik, A. Mohammad Abdul, K. Mogulluru, L. Tarassenko, S. MacMahon, D. Peiris, SMARTHealth India: development and field evaluation of a mobile clinical decision support system for cardiovascular diseases in rural India, JMIR mHealth uHealth 2 (2014) e54, doi:10.2196/mhealth.3568.

[22] D.M.J. Walsh, K. Moran, V. Cornelissen, R. Buys, J. Claes, P. Zampognaro, F. Melillo, N. Maglaveras, I. Chouvarda, A. Triantafyllidis, D. Filos, C.B. Woods, The development and codesign of the PATHway intervention: a theory-driven eHealth platform for the self-management of cardiovascular disease, Transl. Behav. Med. (2018), doi:10.1093/tbm/iby017.

[23] D. Filos, A. Triantafyllidis, I. Chouvarda, R. Buys, V. Cornelissen, W. Budts, D. Walsh, C. Woods, K. Moran, N. Maglaveras, PATHway: decision support in exercise programmes for cardiac rehabilitation, Stud. Health Technol. Inform. 224 (2016) 40-45. http://www.ncbi.nlm.nih.gov/pubmed/27225551. (accessed October 3, 2016)
[24] G. Mancia, R. Fagard, K. Narkiewicz, J. Redon, A. Zanchetti, M. Böhm, T. Chris tiaens, R. Cifkova, G. De Backer, A. Dominiczak, M. Galderisi, D.E. Grobbee, T. Jaarsma, P. Kirchhof, S.E. Kjeldsen, S. Laurent, A.J. Manolis, P.M. Nilsson, L.M. Ruilope, R.E. Schmieder, P.A. Sirnes, P. Sleight, M. Viigimaa, B. Waeber, F. Zannad, ESH/ESC guidelines for the management of arterial hypertension, Blood Press 22 (2013) (2013) 193-278, doi:10.3109/08037051.2013.812549.

[25] A. Chatzitofis, D. Zarpalas, D. Filos, A. Triantafyllidis, I. Chouvarda, N. Maglaveras, P. Daras, Technological module for unsupervised, personalized cardiac rehabilitation exercising, Comput. Softw. Appl. Conf. (COMPSAC), 2017 IEEE 41st Annu., IEEE, Turin, Italy, 2017, doi:10.1109/COMPSAC.2017.230.

[26] F. Ofli, G. Kurillo, S. Obdržálek, R. Bajcsy, H.B. Jimison, M. Pavel, Design and evaluation of an interactive exercise coaching system for older adults: lessons learned, IEEE J. Biomed. Heal. Inform. 20 (2016), doi:10.1109/JBHI.2015. 2391671.

[27] H. Runtti, A. Honka, I. Chouvarda, E. Michail, A. Kokonozi, J. Merilahti, J. Parkaa, M. Gils, Biosignal processing methods to guide cardiac patients to perform safe and beneficial exercise for rehabilitation, Int. J. Bioelectromagn. 15 (2013) $20-25$.

[28] D. Filos, A. Triantafyllidis, V. Manolios, K. Livitckaia, J. Claes, R. Buys, V. Cornelissen, E. Kouidi, N. Maglaveras, I. Chouvarda, Predictive modeling of exercise response in CVD patients under rehabilitation, in: IFMBE Proc., 2018, doi:10.1007/978-981-10-5122-7_51.

[29] L. Richardson, S. Ruby, RESTful Web Services, O'Reilly Media, Inc., 2008.

[30] B. Bonnechère, B. Jansen, P. Salvia, H. Bouzahouene, L. Omelina, F. Moiseev, V. Sholukha, J. Cornelis, M. Rooze, S. Van Sint Jan, Validity and reliability of the Kinect within functional assessment activities: comparison with standard stereophotogrammetry, Gait Posture 39 (2014) 593-598, doi:10.1016/j.gaitpost. 2013.09.018.

[31] S.E. Stahl, H.-S. An, D.M. Dinkel, J.M. Noble, J.-M. Lee, How accurate are the wrist-based heart rate monitors during walking and running activities? Are they accurate enough? BMJ Open Sport Exerc. Med. 2 (2016) e000106, doi:10. 1136/bmjsem-2015-000106.

[32] C.Z. Mooney, Monte Carlo Simulation, 1997

33] C. Luley, A. Blaik, A. Götz, F. Kicherer, S. Kropf, B. Isermann, G. Stumm S. Westphal, Weight loss by telemonitoring of nutrition and physical activity in patients with metabolic syndrome for 1 year, J. Am. Coll. Nutr. 33 (2014), doi: $10.1080 / 07315724.2013 .875437$.

[34] H. Sun, V. De Florio, N. Gui, C. Blondia, Promises and challenges of ambient assisted living systems, in: 2009 Sixth Int. Conf. Inf. Technol. New Gener., IEEE, 2009, pp. 1201-1207, doi:10.1109/ITNG.2009.169.

[35] C. Bryan, S. Boren, The use and effectiveness of electronic clinical decision support tools in the ambulatory/primary care setting: a systematic review of the literature, J. Innov. Heal. Inform. 16 (2008) 79-91, doi:10.14236/jhi.v16i2.679.

[36] A. Wright, D. Sittig, J. Ash, S. Sharma, J. Pang, B. Middleton, Clinical decision support capabilities of commercially-available clinical information systems, J. Am. Med. Inform. Assoc. 16 (2009) 637-644, doi:10.1197/jamia.M3111.

[37] W. Kuijpers, W.G. Groen, N.K. Aaronson, W.H. van Harten, A systematic review of web-based interventions for patient empowerment and physical activity in chronic diseases: relevance for cancer survivors, J. Med. Internet Res. 15 (2013) e37, doi:10.2196/jmir.2281.

[38] A.K. Triantafyllidis, C. Velardo, D. Salvi, S.A. Shah, V.G. Koutkias, L. Tarassenko, A survey of mobile phone sensing, self-reporting and social sharing for pervasive healthcare, IEEE J. Biomed. Heal. Inform. 21 (2017) 218-227, doi:10.1109/ JBHI.2015.2483902.

[39] M. Peleg, Y. Shahar, S. Quaglini, A. Fux, G. García-Sáez, A. Goldstein, M.E. Hernando, D. Klimov, I. Martínez-Sarriegui, C. Napolitano, E. Parimbelli, M. Rigla, L. Sacchi, E. Shalom, P. Soffer, MobiGuide: a personalized and patient-centric decision-support system and its evaluation in the atrial fibrillation and gestational diabetes domains, User Model. User-Adapt. Interact. 27 (2017) 159-213, doi:10.1007/s11257-017-9190-5.

[40] B.Y. Kim, J. Lee, Smart devices for older adults managing chronic disease: a scoping review, JMIR mHealth uHealth 5 (2017) e69, doi:10.2196/mhealth.7141. 